\title{
A TRACE FORMULA OF SPECIAL VALUES OF AUTOMORPHIC L-FUNCTIONS
}

\author{
BERNHARD HEIM
}

\begin{abstract}
Deligne introduced the concept of special values of automorphic L-functions. The arithmetic properties of these L-functions play a fundamental role in modern number theory. In this paper we prove a trace formula which relates special values of the Hecke, Rankin, and the central value of the Garrett triple L-function attached to primitive newforms. This type of trace formula is new and involves special values in the convergent and non-convergent domain of the underlying L-functions.
\end{abstract}

\section{Introduction and Statement of Results}

The main result of this paper is the discovery of an arithmetic trace formula. This formula relates special values of various kinds of automorphic L-functions. Our previous knowledge of the basic facts on the arithmetic nature of special values is built on the fundamental works of some of the pioniers in this field: Siegel [Si69], Klingen [Kl62], Shimura [Sh76], Zagier [Za77], Deligne [De79] and Garrett [Ga87].

Let $g \in S_{k}\left(S L_{2}(\mathbb{Z})\right.$ ) be a primitive (normalized Hecke eigenform) cusp form of integer weight $k$. Let $\left(f_{j}\right)_{j} \in S_{2 k-2}$ and $\left(g_{i}\right)_{i} \in S_{k}$ be primitive eigenbasis. The trace formula compares the weighted average $\sum_{j}$ of special values of the non-trivial piece of the triple L-function $L\left(f_{j} \otimes \operatorname{Sym}^{2}(g), c_{k}\right)$ evaluated at the central value $c_{k}$ and the average $\sum_{i}$ of the triple L-function $L\left(g \otimes g \otimes g_{i}, 2 k-2\right)$ and an error term expressed by special values related to the Rankin L-function attached to $g$. This special value $L\left(f_{j} \otimes \operatorname{Sym}^{2}(g), c_{k}\right)$ and the related triple L-function recently played a prominent role in the proof of the Gross-Prasad conjecture of Saito-Kurokawa lifts given by Ichino [Ich05]. More generally Ikeda stated in [Ik06] a conjecture on the explicit value of a certain period which involves the central value of L-functions (Conjecture 5.1) of the type studied in this paper. There the nonvanishing of the central value is important. Recently some progress has been obtained by Katsurada and Kawamura [KK06]. The focus of this paper is the proof of the arithmetic trace formula and not applications. Nevertheless we believe that there will be applications towards the problems proposed by Iwaniec and Sarnak in the survey article [IS02].

Before we go into more details we put our results into a more general framework and give relations to other results.

2000 Mathematics Subject Classification. 11F.

${ }^{1}$ The author is grateful for the financial support and hospitality of the Max-Planck Institut für Mathematik in Bonn for the last 3 years. The paper contains some extented version of a part of his Habilitationsschrift at the Universität Mannheim. 
Since the eighteenth century since the days of Euler (1707 - 1783) the analytic and arithmetic properties of infinite series of type

$$
L(s):=\sum_{n=1}^{\infty} A(n) n^{-s}(s \in \mathbb{C})
$$

at integral values $m=\ldots-2,-1,0,1,2, \ldots$ have always revealed significant invariants and properties of the underlying motivic object related to the sequence $A(1), A(2), \ldots$ of complex numbers. Significant series arise when the function $A(n)$ is multiplicative and $L(s)$ converges absolutely and locally uniformly if $\operatorname{Re}(s)$ is large enough. These series are nowadays called $L$-functions.

Examples are given by the Dedekind zeta functions $\zeta_{K}(s)$, the Hasse-Weil zeta functions $Z_{E}(s)$ and the Hecke $L(f, s)$ and Rankin L-functions $D(f, s)$ attached to algebraic number fields $K$, elliptic curves $E$ and primitive elliptic cusp forms $f$. They have a meromorphic continuation to the whole complex plane and satisfy a functional equation. Let us just recall some interesting properties. The Riemann zeta function $\zeta(s):=\zeta_{\mathbb{Q}}(s)$ has a single simple pole at $s=1$. The non-vanishing at $\zeta(1+i t)$ for $t \in \mathbb{R}$ directly leads to the prime number theorem. The Kronecker limit formula of $\zeta_{K}$ gives information on the regulator, class number and other invariants of the number field $K$. From Euler we know that

$$
\zeta(2 m)=\frac{(-1)^{m-1} 2^{2 m-1} B_{2 m}}{(2 m) !} \pi^{2 m} \text { for } m \in \mathbb{N} .
$$

Here $B_{m}$ denotes the $m$-th Bernoulli number. Let $\Delta(z)$ be the Ramanujan function, the unique primitive cusp form of level 1 of weight 12, with Fourier coefficients $\tau(n)$. It is known that up to normalization the values of the Rankin type L-function $D(\Delta, s)$ at integral values within the "critical strip" are rational numbers, e.g.,

$$
D(\Delta, 14)=\frac{\zeta(6)}{\zeta(3)} \sum_{n=1}^{\infty} \frac{\tau(n)^{2}}{n^{14}}=\frac{4^{14}}{14 !} \pi^{17}\|\Delta\|^{2} .
$$

Let $\langle$,$\rangle be the Petersson scalar product and \|\|$ the Peterson norm ((see (2.1) for details). Then $\|\Delta\|^{2}=1.03536205679 \times 10^{-6}$ with 12-digits accuracy (see[Za77]).

The concept of critical values of a motivated L-function and conjectures on the arithmetic nature has been introduced by Deligne. Let $\widehat{L}(s):=\gamma(s) L(s)$ be the completion of $L(s)$ at infinity, i.e., $\gamma(s)$ is essentially a product of $\Gamma$-functions with functional equation $\widehat{L}(s)=\widehat{L}(w-s), w \in \mathbb{N}$. Then $m \in \mathbb{Z}$ is a critical value if and only if $\gamma(m)$ and $\gamma(w-m)$ are finite. Deligne preticts that then $L(m)=$ algebraic $\times \Omega_{\text {period. }}$. Moreover a certain functoriality of the action of the automorphism of the absolute Galois group over the involved number fields can be given.

Let $g \in S_{k}$ be primitive with Fourier coefficients $\left(a_{n}(g)\right)_{n}$ and Satake parameter (see 2.2) $\widetilde{\alpha}_{p}, \widetilde{\beta}_{p}$ for all finite prime numbers $p$. For simplification we put

$$
A_{p}(g):=\left(\begin{array}{cc}
\widetilde{\alpha}_{p}(g) & 0 \\
0 & \widetilde{\beta}_{p}(g)
\end{array}\right) .
$$


Then Hecke attached to $g$ the L-function

$$
L(g, s):=\prod_{p}\left\{\operatorname{det}\left(1_{2}-A_{p}(g) p^{-s}\right)\right\}^{-1} \quad \text { for } \operatorname{Re}(s)>\frac{k}{2} .
$$

With this notation the Rankin L-function $D(g, s)$ and the triple L-function $L\left(f_{1} \otimes f_{2} \otimes f_{3}, s\right)$ are defined by

$$
\begin{gathered}
D(g, s):=\zeta(s-k+1)^{-1} \\
\prod_{p}\left\{\operatorname{det}\left(1_{4}-A_{p}(g) \otimes A_{p}(g) p^{-s}\right)\right\}^{-1} \quad \text { for } \operatorname{Re}(s)>k \\
L\left(f_{1} \otimes f_{2} \otimes f_{3}, s\right) \\
:=\prod_{p}\left\{\operatorname{det}\left(1_{8}-A_{p}\left(f_{1}\right) \otimes A_{p}\left(f_{2}\right) \otimes A_{p}\left(f_{3}\right) p^{-s}\right)\right\}^{-1} \quad \text { for } \operatorname{Re}(s) \gg 0 .
\end{gathered}
$$

Here $f_{1}, f_{2}, f_{3}$ are primitive elliptic cusp forms. Let $\widehat{L}(g, s), \widehat{D}(g, s)$ etc. be the completed L-function, see $((2.12)-(2.19))$. They all have a meromorphic continuation to the whole complex plane and satisfy certain functional equations. From this the critical values can explicitly determined. In contrast to the Rankin L-function, the center of the Hecke Lfunction and the triple L-function is always a critical point. The Hecke L-function vanishes in the center if the weight $k$ is congruent to 2 modulo 4 and the triple L-function for the full modular group $S L_{2}(\mathbb{Z})$. This follows from the sign in the functional equation.

Recently a piece of the triple L-function $L\left(f \otimes \operatorname{Sym}^{2}(g), s\right)$ attached to $g \in S_{k}$ and $f \in S_{2 k-2}$ primitive (see (2.10) for a explicit definition) showed up in the proof of the GrossPrasad conjecture of Saito-Kurokawa lifts. Among other things Ichino [Ich05] showed that $L\left(f \otimes \operatorname{Sym}^{2}(g), 2 k-2\right)$ is finite.

More precisely we have the decomposition

$$
L(f \otimes g \otimes g, s)=L\left(f \otimes \operatorname{Sym}^{2}(g), s\right) \cdot L(f, s-k+1) .
$$

Work of Deligne predicts that the unique critical value is given by $2 k-2$ which matches with the center of the functional equation. Now the vanishing of the triple L-function becomes obvious since the Hecke L-function of $f$ vanishes at the center. So it remains an open question to study the arithmetic nature of $L\left(f \otimes \operatorname{Sym}^{2}(g), s\right)$. Ichino [Ich05] proved that the value is zero if and only if a certain period vanishes. Moreover he proved how the special value transforms under the action of any automorphism of $\mathbb{C}$. Recently we have proven [Hei05]: Let $g$ be given then there exists at least one $f$ such that the value

$$
L\left(f \otimes \operatorname{Sym}^{2}(g), 2 k-2\right) \neq 0 .
$$

Here we would like to remark that the opposite is not true. This is not hard to see. One of the main results of this paper is the following: 
Theorem: Arithmetic Trace Formula. Let $k$ be an even positive integer. Let $g \in S_{k}$ be a primitive Hecke eigenform. Then we have

$$
\begin{gathered}
\sum_{i=1}^{\operatorname{dim} S_{2 k-2}} \frac{\widehat{L}\left(f_{i}, 2 k-3\right) \widehat{L}\left(f_{i} \otimes S y m^{2}(g), 2 k-2\right)}{\left\|f_{i}\right\|^{2}\|g\|^{4}} \\
=(-1)^{k / 2} \cdot 2^{k-2} \sum_{j=1}^{\operatorname{dim} S_{k}} \frac{\widehat{L}\left(g \otimes g \otimes g_{j}, 2 k-2\right)}{\|g\|^{4}\left\|g_{j}\right\|^{2}} \\
\quad+\kappa_{1}\left(\frac{\widehat{D}(g, 2 k-2)}{\pi^{\frac{k}{2}-1}\|g\|^{2}}\right)^{2}+\kappa_{2} \frac{\widehat{D}(g, 2 k-2)}{\pi^{\frac{k}{2}-1}\|g\|^{2}} .
\end{gathered}
$$

Here $\left(f_{i}\right)_{i}$ and $\left(g_{j}\right)_{j}$ are primitive Hecke eigenbases of $S_{2 k-2}$ and $S_{k}$ and the constants $\kappa_{1}$ and $\kappa_{2}$ can be explicitly given. We have

$$
\begin{aligned}
& \kappa_{1}=(-1)(-1)^{k / 2} 2^{4} \frac{\Gamma(k)^{2}}{(2 k-2) B_{2 k-2} \Gamma(k / 2)^{2}}, \\
& \kappa_{2}=(-1)(-1)^{k / 2} 2^{2 k+1} \frac{\Gamma(k+1)}{(2 k-2) B_{k} \Gamma(k / 2)} .
\end{aligned}
$$

Remark.

We would like to note that in this paper we actually prove a more general trace formula. It involves the products of roots of $L$-values of type $\widehat{L}\left(f_{i} \otimes \operatorname{Sym}^{2}\left(g_{i_{*}}\right), 2 k-2\right)$ on the left side and the more general triple L-function of type $\widehat{L}\left(g_{i_{1}} \otimes g_{i_{2}} \otimes g_{j}, 2 k-2\right)$ on the right side (see (4.19)). Here $i_{*}=i_{1}$ or $i_{2}$.

Remark.

All the totally real algebraic numbers (see the Subsections 2.1 and 2.3 for more details)

$$
\frac{\widehat{L}\left(f_{i}, 2 k-3\right)}{\Omega_{-}\left(f_{i}\right)}, \frac{\widehat{L}\left(g \otimes g \otimes g_{j}, 2 k-2\right)}{\|g\|^{2}\|g\|^{2}\left\|g_{j}\right\|^{2}} \text { and } \frac{\widehat{D}(g, 2 k-2)}{\pi^{\frac{k}{2}-1}\|g\|^{2}}
$$

are given by evaluating an infinite product, which locally doesn't vanish in the domain of absolute and uniform convergence. Let $f, \ldots, \Phi$ be any Hecke eigenforms, then $K_{f, \ldots, \Phi}$ denotes the field over $\mathbb{Q}$ generated by the corresponding eigenvalues. We put $K_{k}$ if we take all the eigenvalues of an Hecke eigenbasis of $S_{k}$. Then the values given in (1.13) are units in $K_{f_{i}}, K_{g, g_{j}}$ and $K_{g}$. This is not surprising. But new is the fact that these values can be explicitly used to study the central value of the L-function $L\left(f_{i} \otimes \operatorname{Sym}^{2}\left(g_{i_{*}}\right), s\right)$ at the center of symmetry, at least on average.

\section{Automorphic L-Functions}

Let us recall some notation and basic facts on modular forms and L-functions. Moreover we add some properties of Jacobi forms. For the general setting we refer the reader to Iwaniec [Iw97], Eichler and Zagier [EZ85] and Klingen [K190]. Very useful is also the overview article of van der Geer [Ge06]. 
2.1. Basics on L-functions. Let $\mathbb{H}_{g}$ denote the Siegel upper half-space of genus $g$ and let $\Gamma_{g}:=S p_{g}(\mathbb{Z})$ be the Siegel modular group of degree $g$. For $k$ an even non-negative integer let $M_{k}^{(g)}$ be the space of Siegel modular forms of weight $k$ and genus $g$ with respect to $\Gamma_{g}$. Let $S_{k}^{(g)}$ be the subspace of cusp forms. We recall the definition of the Petersson scalar product on $S_{k}^{g}$ :

$$
\langle F, G\rangle:=\int_{\Gamma_{g} \backslash \mathbb{H}_{g}} F(Z) \overline{G(Z)} \operatorname{det}(\operatorname{Im}(Z))^{k+g-1} d Z .
$$

Hence $\|F\|^{2}=\langle F, F\rangle$. To simplify notation we drop the index $g$ in the case $g=1$. Examples of Siegel modular forms are given by Eisenstein series. Let $Z \in \mathbb{H}_{g}$ be an element of the Siegel upper half-space and let $k>g+1$ be even. Then

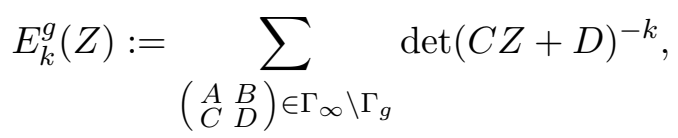

where $\Gamma_{\infty}:=\left\{\left(\begin{array}{cc}A & B \\ 0 & D\end{array}\right) \in \Gamma_{g}\right\}$. This series is absolutely and locally uniformly convergent on $\mathbb{H}_{g}$ and is an element of $M_{k}^{(g)}$. We denote its Fourier coefficients by $A^{E_{k}^{g}}(T)$, where $T \in \mathbb{A}_{g}$ runs through all half-integral symmetric semi-positiv matrices of size $g$. Here $A^{E_{k}^{g}}(0)=1$. Its useful to know that the coefficients $A^{E_{k}^{g}}(T)$ are rational and have bounded denominators. Let $g \in S_{k}$ with Fourier coefficients $\left(a_{n}(g)\right)_{n=1}^{\infty}$. Usually $g$ is called to be primitive if $g$ is a Hecke eigenform and if $a_{1}(g)=1$. Let us assume that $g$ is primitive. Then we attach to every prime number $p$ the local parameters $\widetilde{\alpha}_{p}(g), \widetilde{\beta}_{p}(g) \in \mathbb{C}$ defined by the equations

$$
\widetilde{\alpha}_{p}(g)+\widetilde{\beta}_{p}(g)=a_{p}(g) \text { and } \widetilde{\alpha}_{p}(g) \cdot \widetilde{\beta}_{p}(g)=p^{k-1} .
$$

Then the Satake parameters are given by

$$
\alpha_{p}(g):=p^{-\frac{k-1}{2}} \widetilde{\alpha}_{p}(g) \text { and } \beta_{p}(g):=p^{-\frac{k-1}{2}} \widetilde{\beta}_{p}(g) .
$$

With this notation the Ramanujan Petersson conjecture is usualy found in the literature. It claims that $\left|\alpha_{p}(g)\right|=\left|\beta_{p}(g)\right|=1$ and had been proven by Deligne [De71]. For further simplification we put

$$
A_{p}(g):=\left(\begin{array}{cc}
\widetilde{\alpha}_{p}(g) & 0 \\
0 & \widetilde{\beta}_{p}(g)
\end{array}\right) .
$$

We begin now with the definition of the L-function $L(g, s)$ attached to $g$ of Hecke type. We have the absolute convergent infinite product over all prime numbers

$$
L(g, s):=\prod_{p}\left\{\operatorname{det}\left(1_{2}-A_{p}(g) p^{-s}\right)\right\}^{-1} \quad \text { for } \operatorname{Re}(s)>\frac{k+1}{2} .
$$


The standard L-function $D(g, s)$ or sometimes called the symmetric square L-function of $g$ is given by

$$
\begin{aligned}
D(g, s):= & \zeta(s-k+1)^{-1} \\
& \prod_{p}\left\{\operatorname{det}\left(1_{4}-A_{p}(g) \otimes A_{p}(g) p^{-s}\right)\right\}^{-1} \quad \text { for } \operatorname{Re}(s)>k
\end{aligned}
$$

Here $\zeta(s)$ denotes the Riemann zeta function. These infinite products can also been directly given as Dirichlet series. We have

$$
\begin{aligned}
L(g, s) & =\sum_{n=1}^{\infty} a_{n}(g) n^{-s}, \\
D(g, s) & =\frac{\zeta(2 s-2 k+2)}{\zeta(s-k+1)} \sum_{n=1}^{\infty} a_{n}(g)^{2} n^{-s} .
\end{aligned}
$$

This also explains the name symmetric square.

Let now $f \in S_{2 k-2}$ and $g \in S_{k}$ be primitive. The we put

$$
S_{p}(g):=\left(\begin{array}{ccc}
\widetilde{\alpha}_{p}(g)^{2} & 0 & 0 \\
0 & p^{k-1} & 0 \\
0 & 0 & \widetilde{\beta}_{p}(g)^{2}
\end{array}\right) .
$$

The next L-function $L\left(f \otimes \operatorname{Sym}^{2}(g), s\right)$ is defined by

$$
L\left(f \otimes \operatorname{Sym}^{2}(g), s\right):=\prod_{p}\left\{\operatorname{det}\left(1_{6}-A_{p}(f) \otimes S_{p}(g) p^{-s}\right)\right\}^{-1} \quad \text { for } \operatorname{Re}(s) \gg 0 .
$$

Finally we define the triple L-function. Let $f_{j} \in S_{\nu\left(f_{j}\right)}$ be primitive for $j=1,2,3$. Then we have

$$
\begin{aligned}
L\left(f_{1}\right. & \left.\otimes f_{2} \otimes f_{3}, s\right) \\
& :=\prod_{p}\left\{\operatorname{det}\left(1_{8}-A_{p}\left(f_{1}\right) \otimes A_{p}\left(f_{2}\right) \otimes A_{p}\left(f_{3}\right) p^{-s}\right)\right\}^{-1} \quad \text { for } \operatorname{Re}(s) \gg 0 .
\end{aligned}
$$

All these L-function have a meromorphic continuation to the whole complex s-plane. They also have a functional equation. This can be stated in the "right" way if we add the local factors corresponding to the archimedian prime number with motivic background. Let $\Gamma_{\mathbb{R}}(s):=\pi^{-\frac{s}{2}} \Gamma(s / 2)$ and $\Gamma_{\mathbb{C}}(s):=2(2 \pi)^{-s} \Gamma(s)$ be the normalized $\Gamma$-function. Then we have for $g \in S_{k}$ primitive the completed L-functions

$$
\begin{aligned}
\widehat{L}(g, s) & :=\Gamma_{\mathbb{C}}(s) L(g, s), \\
\widehat{D}(g, s) & :=\Gamma_{\mathbb{R}}(s-k+2) \Gamma_{\mathbb{C}}(s) D(g, s) .
\end{aligned}
$$

Then it is well known that $\widehat{L}(g, s)$ and $D(g, s)$ are entire function on the whole $s$-plane. They have the functional equation

$$
\widehat{L}(g, s)=(-1)^{\frac{k}{2}} \widehat{L}(g, k-s)
$$


and

$$
\widehat{D}(g, s)=\widehat{D}(g, 2 k-1-s) \text {. }
$$

The holomorphic continuation of $D(g, s)$ has first been proven by Shimura. The functional equation had been known already by Rankin. From the times of Hecke the properties of $\widehat{L}(g, s)$ had been known much earlier. Since it is just the Mellin transform of $g$. In the setting of the triple L-function we assume that $\nu\left(f_{1}\right) \geqslant \nu\left(f_{2}\right) \geqslant \nu\left(f_{3}\right)$. Since we are mainly interested in the balanced case we assume that $\nu\left(f_{2}\right)+\nu\left(f_{3}\right) \geqslant \nu\left(f_{1}\right)$. Then

$$
\begin{aligned}
\widehat{L}\left(f_{1} \otimes f_{2} \otimes f_{3}, s\right):= & \Gamma_{\mathbb{C}}(s) \Gamma_{\mathbb{C}}\left(s-\nu\left(f_{1}\right)+1\right) \Gamma_{\mathbb{C}}\left(s-\nu\left(f_{2}\right)+1\right) \\
& \Gamma_{\mathbb{C}}\left(s-\nu\left(f_{3}\right)+1\right) L\left(f_{1} \otimes f_{2} \otimes f_{3}, s\right)
\end{aligned}
$$

This function has a meromorphic continuation to the whole $s$-plane and satisfies the antisymmetric functional equation

$$
\widehat{L}\left(f_{1} \otimes f_{2} \otimes f_{3}, s\right)=-\widehat{L}\left(f_{1} \otimes f_{2} \otimes f_{3}, \nu\left(f_{1}\right)+\nu\left(f_{2}\right)+\nu\left(f_{3}\right)-2-s\right) .
$$

This L-function vanishes in the center $s_{0}=\frac{\nu\left(f_{1}\right)+\nu\left(f_{2}\right)+\nu\left(f_{3}\right)}{2}-1$. Moreover let $f \in S_{2 k-2}$ and $g \in S_{k}$ be primitive. Then we have by a straight forward calculation that

$$
L(f \otimes g \otimes g, s)=L\left(f \otimes \operatorname{Sym}^{2}(g), s\right) \cdot L(f, s-k+1) .
$$

We obtain the following completed L-function

$$
\begin{aligned}
\widehat{L}\left(f \otimes \operatorname{Sym}^{2}(g), s\right) & \\
& :=\Gamma_{\mathbb{C}}(s) \Gamma_{\mathbb{C}}(s-k+1) \Gamma_{\mathbb{C}}(s-2 k+3) L\left(f \otimes \operatorname{Sym}^{2}(g), s\right) .
\end{aligned}
$$

It has a meromorphic continuation to the whole complex $s$-plane and has the functional equation $s \mapsto 4 k-4-s$.

2.2. Saito-Kurokawa correspondance. Let $M_{k-\frac{1}{2}}^{+}\left(\Gamma_{0}(4)\right)$ be Kohnen's plus space. This is the space of modular forms of half-integral weight $k-\frac{1}{2}$ related to the group $\Gamma_{0}(4):=$ $\left\{\left(\begin{array}{ll}a & b \\ c & d\end{array}\right) \in S L_{2}(\mathbb{Z}) \mid c \equiv 0(\bmod 4)\right\}$ where certain Fourier coefficients are zero. Let $S_{k-\frac{1}{2}}^{+}\left(\Gamma_{0}(4)\right)$ be the subspace of cuspforms. Let $J_{k, 1}$ be the space of Jacobi forms of weight $k$ and index 1 and $J_{k, 1}^{\text {cusp }}$ the subspace of cusp forms. Jacobi forms are holomorphic functions on $\mathbb{H} \times \mathbb{C}$ which satiesfy certain conditions (for details see the standard reference [EZ85]).

Let $h_{j} \in S_{k-\frac{1}{2}}^{+}\left(\Gamma_{0}(4)\right)$. Then there exists a Jacobi cuspform $\Phi_{j} \in J_{k, 1}^{\text {cusp }}$ via the isomorphism given in Theorem 5.4 in ([EZ85]. This isomorphism is given on the level of Fourier coefficients and is compatible with the action of the Hecke algebra of Jacobi forms and modular forms of half-integral weight. Let $(\lambda(n))_{n}$ be the eigenvalues. Then $f(z)=\sum_{n} \lambda(n) e^{2 \pi i n z} \in S_{2 k-2}$ is a primitive Hecke eigenform. This is the Shimura isomorphism.

Moreover these spaces are isomorphic to the (cuspidal) Maass Spezialschar, a certain subspace of $S_{k}^{(2)}$. Let further $\langle\rangle,,\langle,\rangle_{J}$ and $\langle,\rangle_{+}$denote the Petersson scalar products on $M_{k}^{(g)}$, the space of Jacobi forms and the plus space. Moreover let \|\|$_{*}$ be the related 
Petersson norm.

Let $g \in S_{k}$ be primitive. Then we denote by $K_{g}$ the field generated by the eigenvalues of $g$. It is well known that $K_{g}$ is a totally real number field. Let finitely many Hecke eigenforms $f_{1}, \cdots f_{l}$ be given. They can be Siegel modular forms, Jacobi forms or modular forms of half-integral weight. Then we denote by $K_{f_{1}, \ldots, f_{l}}$ the field generated by the eigenvalues. Let $f \in S_{2 k-2}$ primitive be given. Then we can choose $h \in S_{k-\frac{1}{2}}^{+}\left(\Gamma_{0}(4)\right)$ via the Shimura correspondance such that the Fourier coefficients are all contained in $K_{f}$. Similary we can choose the related Jacobi form $\Phi$. Such $h$ and $\Phi$ we call normalized.

\subsection{Algebraicity of critical values of automorphic L-functions.}

The general philosophy of Deligne [De79] predicts for any "motivated" Dirichlet series $L(s)$ the structure of the arithmetic nature of certain "critical" values. The underlying assumption is that the Dirichlet series arise from some algebraic variety, Galois representation or modular form and have a functional equation of the form

$$
\widehat{L}(s)=\gamma(s) L(s)=\varepsilon \widehat{L}(w-s), \quad \varepsilon \text { is root of unity, } w \text { is a constant }
$$

and $\gamma(s)$ is a $\Gamma$-factor. Then all integers $m$ for which $\gamma(m)$ and $\gamma(w-m)$ is finite is denoted (special) critical value. It is expected that $L(m)=$ algebraic $\times \Omega$, where $\Omega$ is a period "on which something nice can be said" (Don Zagier).

\section{a) Hecke L-function $L(g, s)$}

Let $g \in S_{k}$ be primitive. Then the critical values of the L-function $L(g, s)$ are given by the integers $m=1,2, \ldots, k-1$. We want also to remark that the center $m_{0}=k / 2$ is also a critical value and $L\left(g, m_{0}\right)=0$ if $k \equiv 2(\bmod 4)$. We know from the result of Eichler-Shimura-Manin that there exist two periods $\Omega_{-}(g), \Omega_{+}(g) \in \mathbb{R}$ such that for the critical values $m=\frac{k}{2}, \ldots, k-1$ we have

$$
\frac{\widehat{L}(g, m)}{\Omega_{(-1)^{m}}(g)} \in K_{g} .
$$

Here we identify $(-1)^{k}$ with + or - in the obvious way. The explicit nature of the other critical values follows directly from the functional equation (see also [Ge06],§26).

b) Rankin L-function $D(g, s)$

Let $g \in S_{k}$ be primitive. Then the critical values of the Rankin type L-function $D(g, s)$ are given by $m=1,3, \ldots, k-1$ and $k, k+2, \ldots, 2 k-2$. Here the center $m_{0}=\frac{2 k-2}{2}$ is not an integer and hence not a critical value. We have

$$
\frac{D(g, m)}{\pi^{2 m-k+1}\|g\|^{2}}=\left(2^{1-m} \Gamma\left(\frac{m-k+2}{2}\right) \Gamma(m)\right)^{-1} \pi^{\frac{k-m}{2}} \frac{\widehat{D}(g, m)}{\|g\|^{2}} \in K_{g}
$$

for the even critical values. Supplementary we deduce from the funtional equation, that for the odd critical values we have $D(g, m) /\left(\pi^{m}\|g\|^{2}\right) \in K_{g}$. 


\section{c) Triple L-function}

For the triple L-function $L\left(f_{1} \otimes f_{2} \otimes f_{3}, s\right)$ with $f_{j} \in S_{\nu_{j}}$ we fix the ordering $\nu\left(f_{1}\right) \geqslant$ $\nu\left(f_{2}\right) \geqslant \nu\left(f_{3}\right)$ and assume that we are in the situation of the balanced case $\nu\left(f_{2}\right)+\nu\left(f_{3}\right) \geqslant$ $\nu\left(f_{1}\right)$. Then the critical values $m$ are given by

$$
\nu\left(f_{1}\right) \leqslant m \leqslant \nu\left(f_{2}\right)+\nu\left(f_{3}\right)-2 .
$$

Here the center $m_{0}=\frac{\nu\left(f_{1}\right)+\nu\left(f_{2}\right)+\nu\left(f_{3}\right)}{2}-2$ is also a critical value. It can deduced from the functional equation and the some finiteness theorem that the triple L-function vanishes in the center (see Orloff ([Or87]). Moreover we have

$$
\frac{L\left(f_{1} \otimes f_{2} \otimes f_{3}, m\right)}{\pi^{4 m+A}\left\|f_{1}\right\|^{2}\left\|f_{2}\right\|^{2}\left\|f_{3}\right\|^{2}} \in K_{f_{1}, f_{2}, f_{2}},
$$

with $A=3-\nu\left(f_{1}\right)-\nu\left(f_{2}\right)-\nu\left(f_{3}\right)$.

EXAMPLE: Let $f_{1}=f \in S_{2 k-2}$ and $f_{2}=f_{3}=g \in S_{k}$ be primitive. Then we have exactly one critical value $m=2 k-2$. This is also equal to the center. Hence we have $L(f \otimes g \otimes g, 2 k-2)=0$. Moreover let $m=2 k-2$ be a critical value and let $\nu\left(f_{1}\right)=\nu\left(f_{2}\right)=\nu\left(f_{3}\right)=k$. Then we have

$$
\frac{\widehat{L}\left(f_{1} \otimes f_{2} \otimes f_{3}, 2 k-2\right)}{\left\|f_{1}\right\|^{2}\left\|f_{2}\right\|^{2}\left\|f_{3}\right\|^{2}} \in K_{f_{1}, f_{2}, f_{2}} .
$$

d) L-function $L\left(f \otimes \mathbf{S y m}^{2}(g), s\right)$

Let $f \in S_{2 k-2}$ and $g \in S_{k}$ be primitive. Then the critical values of the L-function $L\left(f \otimes \operatorname{Sym}^{2}(g), s\right)$ is given by one number $m=2 k-2$. Moreover we have

$$
\frac{\widehat{L}\left(f \otimes \operatorname{Sym}^{2}(g), 2 k-2\right)}{\Omega_{+}(f)\|g\|^{4}} \in K_{f, g} .
$$

(See also Ichino [Ich05] for details).

\section{Numerical verification of the Trace Formula}

We consider the Arithmetic Trace Formula stated in the introduction for the weight $k=12$ and choose the unique primitive Hecke eigenforms Let $\Delta \in S_{12}$ and $f \in S_{22}$ be the unique primitive Hecke eigenforms of weight 12 and 22. Then we have

$$
\begin{array}{lrl}
\Delta(z)= & q-24 q^{2}+252 q^{3}-1472 q^{4}+4830 q^{5}-6048 q^{6}+\ldots & =\sum_{n=1}^{\infty} \tau(n) q^{n} \\
f(z)= & q-288 q^{2}-128844 q^{3}-2014208 q^{4}+21640950 q^{5}+\ldots & =\sum_{n=1}^{\infty} b(n) q^{n}
\end{array}
$$

The Petersson norm of a Hecke eigenform $g \in S_{k}$ can be identified with a special value of the standard zeta function $D(g, s)$ of $g$ ([Za77], (5)), this is due to Rankin. The 
correspondance is given by

$$
\|g\|^{2}=\frac{(k-1) !}{2^{2 k-1} \pi^{k+1}} D(g, k) .
$$

The special value $D(g, k)$ can be determined by meromorphic continuation. There is a useful programm of Dokchister [Do04] to calculate such values. This leads to

$$
\begin{aligned}
\|\Delta\|^{2} & =0.00000103536205680432092234 \ldots \\
\|f\|^{2} & =0.00002009981832327430645231 \ldots
\end{aligned}
$$

Our first goal is to determine the numerical value of the left side of the trace formula. The value of $\widehat{L}\left(f \otimes \operatorname{Sym}^{2}(\Delta), 22\right)$ can again be determined with the programm of Dokchister (see also Ichino [Ich05]). We have

$$
\begin{aligned}
L(f, 23) & =0.99988499414258382599524516 \ldots \\
\widehat{L}(f, 23) & =84.2000215244544365950065601 \ldots \\
\widehat{L}\left(f \otimes \operatorname{Sym}^{2}(\Delta), 22\right) & =0.75704862297802829562086575 \ldots
\end{aligned}
$$

Hence

$$
\sum_{i=1}^{\operatorname{dim} S_{2 k-2}} \frac{\widehat{L}\left(f_{i}, 2 k-3\right) \widehat{L}\left(f_{i} \otimes \operatorname{Sym}^{2}(g), 2 k-2\right)}{\left\|f_{i}\right\|^{2}\|g\|^{4}}
$$

for $k=12$ is equal to the numerical value

$$
2958416757652464643.22953541 \ldots
$$

This number has been obtained directly. From the proof of the trace formula we know that this number should actually be a rational number. A careful analysis leads to the candidate

$$
\frac{2^{56} \cdot 3^{6} \cdot 5^{4} \cdot 7}{131 \cdot 593}
$$

which coincides with $2958416757652464643.22953541 \ldots$ in the range of precision.

On the right side we first determine the value of

$$
\frac{\widehat{D}(g, 2 k-2)}{\pi^{\frac{k}{2}-1}\|g\|^{2}}
$$

for $g=\Delta$. We obtain directly

$$
D(\Delta, 22)=0.99964571112477139783572962 \ldots
$$

and hence

$$
\frac{\widehat{D}(\Delta, 22)}{\pi^{5}\|\Delta\|^{2}}=110841.734096772163845718240 \ldots
$$


The constants $\kappa_{0}, \kappa_{1}, \kappa_{2}$ for $k=12$ :

$$
\begin{aligned}
& \kappa_{0}(k)=(-1)^{k / 2} 2^{k-2}, \\
& \kappa_{1}(k)=(-1)(-1)^{k / 2} 2^{4} \frac{\Gamma(k)^{2}}{(2 k-2) B_{2 k-2} \Gamma(k / 2)^{2}}, \\
& \kappa_{2}(k)=(-1)(-1)^{k / 2} 2^{2 k+1} \frac{\Gamma(k+1)}{(2 k-2) B_{k} \Gamma(k / 2)} .
\end{aligned}
$$

are explicity given by

$$
\begin{aligned}
& \kappa_{0}=10240=2^{10} \\
& \kappa_{1}=-12995908.891263210741088 \ldots=\frac{(-1) \cdot 2^{14} \cdot 3^{7} \cdot 5^{2} \cdot 7^{2} \cdot 23}{131 \cdot 593} \\
& \kappa_{2}=24052904584483.936324167872648 \ldots=\frac{2^{32} \cdot 3^{5} \cdot 5^{2} \cdot 7^{2} \cdot 13}{691} .
\end{aligned}
$$

The special value of the triple L-function $L(\Delta \otimes \Delta \otimes \Delta, s)$ at $s=22$ we determine via the local factors of the Euler product by calculating the Satake parameters of $\Delta$. Hence we obtain

$$
L(\Delta \otimes \Delta \otimes \Delta, 22)=0.99602837097824593011931492 \ldots .
$$

Then we obtain for $k=12$ :

$$
\sum_{j=1}^{\operatorname{dim} S_{k}} \frac{\widehat{L}\left(g \otimes g \otimes g_{j}, 2 k-2\right)}{\|g\|^{4}\left\|g_{j}\right\|^{2}}
$$

is equal to

$$
441423252695906.208342030317 \ldots .
$$

So finally we have for the expression

$$
\kappa_{0} \cdot \frac{\widehat{L}(\Delta \otimes \Delta \otimes \Delta, 22)}{\|\Delta\|^{6}}+\kappa_{1} \cdot\left(\frac{\widehat{D}(\Delta, 22)}{\pi^{5}\|\Delta\|^{2}}\right)^{2}+\kappa_{2} \cdot \frac{\widehat{D}(\Delta, 22)}{\pi^{5}\|\Delta\|^{2}}
$$

the explicit value

$$
2958416757652464643.22111654 \ldots \text {. }
$$

This shows that the Arithmetic Trace formula for the weight $k=12$ can be numerically verified.

\section{Proof of the Arithmetic Trace Formula}

This section is devoted to the Arithmetic Trace Formula stated in the introduction. We give a proof which is constructive and explicit. Moreover as already remarked we give a more general formula which may be useful for further applications. 
Proof. We prove the theorem with an extension of a technique related to the doubling method in the setting of modular and Jacobi forms. There the so called big cell plays a fundamental role. It is related to the unique non-negligible orbit which leads to an integral representation of an automorphic L-function. For our purpose it is not enough to know one orbit we need them all. Actually we need the whole pullback formula related to the orbits. What does this mean? Let us fix the diagonal embeding $\mathbb{H} \times \mathbb{H} \hookrightarrow \mathbb{H}_{2}$. Here

$$
(Z, W) \mapsto\left(\begin{array}{cc}
Z & 0 \\
0 & W
\end{array}\right)
$$

This can be generalized in the obvious way to an embedding $\mathbb{H} \times \mathbb{H} \times \mathbb{H} \hookrightarrow \mathbb{H}_{3}$. Let $\left(g_{j}\right)_{j}$ be a Hecke eigenbasis of $S_{k}$ with $a_{1}\left(g_{j}\right)=1$, i.e., $g_{j}$ is assumed to be primitive. This always exists. Garrett [Ga84] has discovered the following beautiful formula:

$$
\left.E_{k}^{(2)}\right|_{\mathbb{H} \times \mathbb{H}}=E_{k} \otimes E_{k}+\sum_{j=1}^{\operatorname{dim} S_{k}} d_{j} g_{j} \otimes g_{j} .
$$

It had been well known since the time of Witt that the restriction of a modular form of genus $n$ on blocks of size $n_{1}+\cdots+n_{l}=n$ is an element of $M_{k}^{\left(n_{1}\right)} \otimes \cdots \otimes M_{k}^{\left(n_{l}\right)}$. That the image in the case $n=2$ is contained in the "diagonal" of a Hecke eigenbasis was surprising. Most important is that the numbers $d_{j}$ have a significant arithmetic meaning. They are related to a critical value of the Rankin L-function. From this we can deduce that these numbers are elements of $K_{g_{j}}$ and are not zero. They can be explicitly determined:

$$
d_{j}=\frac{(-1)^{\frac{k}{2}} 2^{3-k} \pi D\left(g_{j}, 2 k-2\right)}{(k-1) \zeta(k) \zeta(2 k-2)\left\|g_{j}\right\|^{2}} .
$$

The situation in the case $3=1+1+1$ if different. Garrett [Ga87] computed the scalar product of the restricted Eisenstein series with three elliptic cusp forms. A detailed analysis and combination of the two papers of Garrett (see also [He99]) leads to the complete pullback formula. We obtain:

$$
\begin{aligned}
E_{k}^{(3)} \mid \mathbb{H} \times \mathbb{H} \times \mathbb{H} & =E_{k} \otimes E_{k} \times E_{k}+\sum_{j=1}^{\operatorname{dim} S_{k}} d_{j} E_{k} \times g_{j} \otimes g_{j} \\
& +\sum_{j=1}^{\operatorname{dim} S_{k}} d_{j} g_{j} \otimes E_{k} \otimes g_{j}+\sum_{j=1}^{\operatorname{dim} S_{k}} d_{j} g_{j} \otimes g_{j} \otimes E_{k} \\
& +\sum_{i, j, m=1}^{\operatorname{dim} S_{k}} l_{i, j, m} g_{i} \otimes g_{j} \otimes g_{m} .
\end{aligned}
$$

Here we have $l_{i, j, m} \in K_{g_{i}, g_{j}, g_{m}}^{\times}$, the composition field of $K_{g_{i}}, K_{g_{j}}, K_{g_{m}}$. These numbers are essentially critical values of the triple L-function in the sense of Deligne. They had been first explicitely determined by Garrett [Ga87] (see also Mizumoto [Mi97], page 192, and 
Heim [He99], page 236, for the explicit value of the constants and further explanation):

$$
\begin{aligned}
l_{i, j, m}= & (-1)^{\frac{k}{2}} \cdot 2^{-5 k+8} \frac{\Gamma(k-1)^{3}}{\Gamma(k)} \\
& \times \frac{\pi^{3-2 k} L\left(g_{i} \otimes g_{j} \otimes g_{m}, 2 k-2\right)}{\zeta(2 k-2) \zeta(k)\left\|g_{i}\right\|^{2}\left\|g_{j}\right\|^{2}\left\|g_{m}\right\|^{2}} .
\end{aligned}
$$

Here we would like to remark that all three cusp forms have the same weight. For a more general formula allowing also different weights one has has to use differential operators. Moreover the big cell is related to

$$
\sum_{i, j, m=1}^{\operatorname{dim} S_{k}} l_{i, j, m} g_{i} \otimes g_{j} \otimes g_{m} .
$$

But we will see imediately that one also needs one of the negligible orbits for the trace formula.

The next step is to extract the first coefficient of the Fourier expansion with respect to the third variable. It is important that this procedure is the same as starting with a Fourier-Jacobi expansion of the involved Siegel Eisenstein series, then extracting the the first coefficient and then restrict the domain $\mathbb{H}_{2} \times \mathbb{C}^{2}$ to $\mathbb{H} \times \mathbb{H}$. Let $B_{k}$ be the $k$-th Bernoulli number. Then we have

$$
\begin{array}{r}
\frac{-2 k}{B_{k}} E_{k} \otimes E_{k}+\sum_{j=1}^{\operatorname{dim} S_{k}} d_{j} E_{k} \otimes g_{j}+\sum_{j=1}^{\operatorname{dim} S_{k}} d_{j} g_{j} \otimes E_{k} \\
+\frac{-2 k}{B_{k}} \sum_{j=1}^{\operatorname{dim} S_{k}} d_{j} g_{j} \otimes g_{j}+\sum_{i, j, m=1}^{\operatorname{dim} S_{k}} l_{i, j, m} g_{i} \otimes g_{j} .
\end{array}
$$

Here we would like to mention that it turns out to be very convinient to have normalized our Siegel Eisenstein series, such that the 0-th coefficient is always one, since it is compatible with restricting Eisenstein series to the diagonal.

Let $\delta_{i, j}=1$ if $i=j$ and 0 otherwise. Then the coefficient of the basis element $g_{i} \otimes g_{j} \in$ $S_{k} \otimes S_{k}$ is given by

$$
\delta_{i j} d_{j} \cdot \frac{-2 k}{B_{k}}+\sum_{m=1}^{\operatorname{dim} S_{k}} l_{i, j, m} \text {. }
$$

Now we do something which we haven't found yet in the literature. We determine a second pullback formula of our Eisenstein series, with respect to a not obvious embeding of the Jacobi spaces $\mathbb{H}^{J} \times \mathbb{H}^{J}$ into $\mathbb{H}_{3}$ and obtain something new. Here $\mathbb{H}^{J}:=\mathbb{H} \times \mathbb{C}$. We start by looking directly at the Fourier-Jacobi expansion of the Eisenstein series of genus 3 . It is convenient to parametrize elements of $\mathbb{H}_{3}$ in the following way:

$$
Z=\left(\begin{array}{ccc}
\tau_{1} & z & z_{1} \\
z & \tau_{2} & z_{2} \\
z_{1} & z_{2} & \tau_{3}
\end{array}\right)
$$


We fix the diagonal embeding $\mathbb{H}^{J} \times \mathbb{H}^{J} \hookrightarrow \mathbb{H}_{3}$ given by

$$
\left(\tau_{1}, z_{1}\right),\left(\tau_{2}, z_{2}\right) \mapsto\left(\begin{array}{ccc}
\tau_{1} & 0 & z_{1} \\
0 & \tau_{2} & z_{2} \\
z_{1} & z_{2} & \tau_{3}
\end{array}\right) .
$$

With this notation the Fourier-Jacobi expansion of $E_{k}^{(3)}(Z)$ with respect to $\tau_{3}$ is given by

$$
E_{k}^{(3)}(Z)=\sum_{n=0}^{\infty} e_{k, n}^{(3)}\left(\left(\begin{array}{cc}
\tau_{1} & z \\
z & \tau_{2}
\end{array}\right),\left(z_{1}, z_{2}\right)\right) e^{2 \pi i n \tau_{3}} .
$$

The Fourier-Jacobi coefficients are Jacobiforms on $\mathbb{H}_{2} \times \mathbb{C}^{2}$ of weight $k$ and index $n$. By switching to Jacobi Eisenstein series and having a "compatible" normalization we normalize the Jacobi Eisenstein series in such a way that the 0-th Fourier coefficient is equal to 1 . In this case we have

$$
E_{k, n}^{J, 2}\left(\left(\underset{z}{\tau_{1}} \underset{\tau_{2}}{z}\right),\left(z_{1}, z_{2}\right)\right)=\frac{B_{k}}{-2 k \sigma_{k-1}(n)} e_{k, n}^{(3)}\left(\left(\begin{array}{cc}
\tau_{1} & z \\
z & \tau_{2}
\end{array}\right),\left(z_{1}, z_{2}\right)\right)
$$

Here $\sigma_{k-1}(n):=\sum_{d \mid n} d^{k-1}$. Let $\left(\Phi_{j}\right)_{j}$ be a normalized Hecke eigenbasis of $J_{k, 1}^{\text {cusp }}$, i.e., a Hecke eigenbasis such that all the Fourier coefficients are contained in the field $K_{\Phi_{j}}$ generated by all the eigenvalues. Let $f_{j} \in S_{2 k-2}$ be primitive and correspond to $\Phi_{j}$ via the Shimura correspondance. Then $\left(f_{j}\right)_{j}$ is a Hecke eigenbasis of $S_{2 k-2}$ with the same eigenvalues. Obviously we have $K_{f_{j}}=K_{\Phi_{j}}$. Arakawa [Ar94] found out that also in the setting of Jacobi forms the doubling method has a certain interpretation. But it turned out that the underlying Hecke-Jacobi theory is much more complicated as expected [AH98], [He01]. But anyway some results can be obtained. We deduce from [Ar94]:

$$
\left.E_{k, 1}^{J, 2}\right|_{\mathbb{H}^{J} \times \mathbb{H}^{J}}=E_{k, 1}^{J} \otimes E_{k, 1}^{J}+\sum_{m=1}^{\operatorname{dim} J_{k, 1}^{\text {cusp }}} \alpha_{m} \Phi_{m} \otimes \Phi_{m} .
$$

Here $E_{k, 1}^{J}$ is the Jacobi Eisenstein series of weight $k$ and index 1 on $\mathbb{H} \times \mathbb{C}$ as introduced in [EZ85]. The numbers $\alpha_{j}$ are related with the critical values of the Hecke L-function attached to $f_{j}$. We have:

$$
\alpha_{m}=\frac{(-1)^{k / 2} \pi 2^{1-k}}{(k-3 / 2)} \frac{L\left(f_{m}, 2 k-3\right)}{\left\|\Phi_{m}\right\|^{2} \zeta(2 k-2)} .
$$

For details see [Ar94] and [He01]. Since up to normalization $E_{k, 1}^{J}$ is the first Fourier-Jacobi coefficient of $E_{k}^{(2)}$ and these Eisenstein series are in the Maass Spezialschar we have

$$
\left.E_{k, 1}^{J}\right|_{\mathbb{H}}=E_{k}+\frac{B_{k}}{-2 k} \sum_{j=1}^{\operatorname{dim} S_{k}} d_{j} g_{j}
$$

This formula can be deduced from the fact that the Siegel Eisenstein series of genus 2 is an element from the so called Maass Spezialschar. It is then an easy exercise to obtain 
the formula. Further we have formaly that

$$
\left.\Phi_{m}\right|_{\mathbb{H}}=\sum_{j=1}^{\operatorname{dim} S_{k}} \gamma_{j}^{m} g_{j} .
$$

From the arithmetic of the Fourier coefficients of the Jacobi form we can deduce that $\gamma_{j}^{m}$ are totally real algebraic numbers. Let now $h_{m}$ be the modular form of half-integral weight directly related to the Jacobi form $\Phi_{m}$ via the isomorphism given in [EZ85], Theorem 5.4 (see also Subsection 2.2). Then we can combine Proposition 4.3 given in [He98] and the explicit description of Ichino [Ich05] of the square of the pullback of a Saito-Kurokawa lift. Again by a straightforward calculation we get

$$
\left(\gamma_{j}^{m}\right)^{2}=2^{-k} \frac{\left\|h_{m}\right\|^{2}}{\left\|f_{m}\right\|^{2}\left\|g_{j}\right\|^{4}} \widehat{L}\left(f_{m} \otimes \operatorname{Sym}^{2}\left(g_{j}\right), 2 k-2\right) .
$$

Hence we obtain for the coefficient of $g_{i} \otimes g_{j}$ in the pullback formula of $\left.\frac{-2 k}{B_{k}} E_{k, 1}^{J, 2}\right|_{\mathbb{H} \times \mathbb{H}}$ the expression

$$
\frac{B_{k}}{-2 k} d_{i} \cdot d_{j}+\frac{-2 k}{B_{k}} \sum_{m=1}^{\operatorname{dim} S_{2 k-2}} \alpha_{m} \gamma_{i}^{m} \gamma_{j}^{m} .
$$

In the next step we compare the two pullback formulas one in the setting of modular forms and the other deduced from the work of Arakawa in the setting of Jacobi forms. This leads to

$$
\delta_{i j} d_{j} \cdot \frac{-2 k}{B_{k}}+\sum_{m=1}^{\operatorname{dim} S_{k}} l_{i, j, m}=\frac{B_{k}}{-2 k} d_{i} \cdot d_{j}+\frac{-2 k}{B_{k}} \sum_{m=1}^{\operatorname{dim} S_{2 k-2}} \alpha_{m} \gamma_{i}^{m} \gamma_{j}^{m} .
$$

This formula is the heart of our approach. It contains much more informations as we use at the moment. To prove the trace formula we restrict ourself to the case $i=j$. We want to mention that if $i \neq j$, then on one side the formula simplifies because the summand $\delta_{i j} d_{j} \cdot \frac{-2 k}{B_{k}}$ disappears. But on the other side we only know the value of $\left(\gamma_{i}^{m}\right)^{2}$ which is totally real algebraic number. So still the delicate question of the sign of the root remains open. Nevertheless we obtain from (2.13) and (4.3) the explicit formula

$$
d_{j}=-2^{5-2 k} \cdot \frac{\Gamma(k+1)}{\Gamma(k / 2)} \cdot \frac{1}{B_{k} B_{2 k-2}} \cdot \frac{\widehat{D}\left(g_{j}, 2 k-2\right)}{\pi^{\frac{k}{2}-1}\left\|g_{j}\right\|^{2}} .
$$

Moreover from (2.16) and (4.5) we obtain

$$
l_{j, j, m}=-2^{3-3 k} \cdot \frac{k \cdot(2 k-2)}{B_{k} B_{2 k-2}} \frac{\widehat{L}\left(g_{j} \otimes g_{j} \otimes g_{m}, 2 k-2\right)}{\left\|g_{j}\right\|^{2}\left\|g_{j}\right\|^{2}\left\|g_{m}\right\|^{2}} .
$$

And from (2.12) and (4.14) we obtain

$$
\alpha_{j}=(-1)^{\frac{k}{2}} 2^{1-k} \cdot \frac{2 k-2}{B_{2 k-2}} \frac{\widehat{L}\left(f_{j}, 2 k-3\right)}{\left\|\Phi_{j}\right\|^{2}} .
$$

Let $h_{j} \in S_{k-\frac{1}{2}}^{+}\left(\Gamma_{0}(4)\right)$ be normalized and related to $\Phi_{j} \in J_{k, 1}^{\text {cusp }}$ via the isomorphism given in Theorem 5.4 in ([EZ85]. Then we obtain for example from ([KS89], §2), the 
transformation law for the square of the norms given by $\left\|\Phi_{j}\right\|^{2}=2^{2 k-3}\left\|h_{j}\right\|^{2}$. This leads to

$$
\alpha_{j}=(-1)^{\frac{k}{2}} 2^{4-3 k} \cdot \frac{2 k-2}{B_{2 k-2}} \frac{\widehat{L}\left(f_{j}, 2 k-3\right)}{\left\|h_{j}\right\|^{2}} .
$$

Then we have

$$
\alpha_{j}\left(\gamma_{j}^{m}\right)^{2}=\kappa \frac{\widehat{L}\left(f_{m}, 2 k-3\right) \widehat{L}\left(f_{m} \otimes \operatorname{Sym}^{2}\left(g_{j}\right), 2 k-2\right)}{\left\|f_{m}\right\|^{2} \cdot\left\|g_{j}\right\|^{4}} .
$$

Here $\kappa=(-1)^{\frac{k}{2}} 2^{4-4 k} \frac{2 k-2}{B_{2 k-2}}$. If we summarize everthing and plugging into (4.19) this leads to

$$
\begin{gathered}
-\frac{-2 k}{B_{k}} \frac{\Gamma(k+1)}{\Gamma(k / 2)} \cdot \frac{2^{5-2 k}}{B_{k} B_{2 k-2}} \frac{\widehat{D}\left(g_{j}, 2 k-2\right)}{\pi^{\frac{k}{2}-1}\left\|g_{j}\right\|^{2}}-2^{3-3 k} \frac{k \cdot(2 k-2)}{B_{k} B_{2 k-2}} \sum_{t=1}^{\operatorname{dim} S_{k}} \frac{\widehat{L}\left(g_{j} \otimes g_{j} \otimes g_{t}, 2 k-2\right)}{\left\|g_{j}\right\|^{2}\left\|g_{j}\right\|^{2}\left\|g_{t}\right\|^{2}} \\
=\frac{B_{k}}{-2 k} 2^{10-4 k} \frac{\Gamma(k+1)^{2}}{\Gamma(k / 2)^{2}} \cdot \frac{1}{B_{k}^{2} B_{2 k-2}^{2}}\left(\frac{\widehat{D}\left(g_{j}, 2 k-2\right)}{\pi^{\frac{k}{2}-1}\left\|g_{j}\right\|^{2}}\right)^{2} \\
\quad+(-1)^{\frac{k}{2}} \frac{-2 k}{B_{k}} 2^{4-4 k} \frac{2 k-2}{B_{2 k-2}} \sum_{m=1}^{\operatorname{dim} S_{2 k-2}} \frac{\widehat{L}\left(f_{m}, 2 k-3\right) \widehat{L}\left(f_{m} \otimes \operatorname{Sym}^{2}\left(g_{j}\right), 2 k-2\right)}{\left\|f_{m}\right\|^{2} \cdot\left\|g_{j}\right\|^{4}} .
\end{gathered}
$$

Finally we obtain by a straightforward calculation the desired result.

\section{REFERENCES}

[Ar94] T. Arakawa: Jacobi Eisenstein series and a basis problem for Jacobi forms. Comm. Mathematici Universitatis Sancti Pauli. 43 (1994), 181-216.

[AH98] T. Arakawa, B. Heim: Real analytic Jacobi Eisenstein series and Dirichlet series attached to three Jacobi forms. Max-Planck-Institut Bonn. Series 66 (1998)

[Bo85] S. Böcherer: Über die Funktionalgleichung automorpher L-Funktionen zur Siegelschen Modulgruppe. J. reine angew. Math. 362 (1985), 146-168.

[De71] P. Deligne: Formes modulaires et representations l-adic. Lect. Notes Math. 179 (1971), BerlinHeidelberg-New York, 139-172.

[De79] P. Deligne: Valeurs de fonctions L et periode d'integrales. Proc. Symposia Pure Math. 33 (1979), part 2, 313-346.

[Do04] T. Dokchitser: Computing special values of motivic L-functions. Exp. Math. 13 (2004), 137-149.

[EZ85] M. Eichler, D. Zagier: The theory of Jacobi forms. Progress in Mathematics. Vol. 55. BostonBasel-Stuttgart: Birkhäuser (1985).

[Ga84] P. Garrett: Pullbacks of Eisenstein series; applications. Automorphic forms of several variables (Katata, 1983), 114-137, Progr. Math., 46 Birkhäuser Boston, Boston, MA, 1984.

[Ga87] P. Garrett: Decomposition of Eisenstein series: triple product L-functions. Ann. Math. 125 (1987), 209-235.

[Ge06] G. van der Geer: Siegel modular forms. manuscript on arXiv:math.AG/0605346 v1

[He98] B. Heim: Über Poincare Reihen und Restriktionsabbildungen. Abh. Math. Sem. Univ. Hamburg. 68 (1998), 79-89 
[He99] B. Heim: Pullbacks of Eisenstein series, Hecke-Jacobi theory and automorphic L-functions. In: Automorphic Froms, Automorphic Representations and Arithmetic. Proceedings of Symposia of Pure Mathematics 66, part 2 (1999).

[He01] B. Heim: L-functions for Jacobi forms and the basis problem. Manuscripta Math. 106 (2001), 489-503.

[Hei05] B. Heim: On injectivity of the Satoh lifting of modular forms and the Taylor coefficients of Jacobi forms. Preprint MPI-Bonn Series 68, 2005

[Hei06] B. Heim: Period integrals and the global Gross-Prasad conjecture. Preprint 2006

[Ich05] A. Ichino: Pullbacks of Saito-Kurokawa Lifts. Invent. Math. 162 (2005), 551-647.

[Ik01] T. Ikeda: On the lifting of elliptic cusp forms to Siegel cusp forms of degree 2n. Ann. of Math. 154 no. 3 (2001), 641-681.

[Ik06] T. Ikeda: Pullback of the lifting of elliptic cusp forms and Miyawakis conjecture. Duke Math. Journal, 131 no. 3 (2006), 469-497.

[Iw97] H. Iwaniec: Topics in classical automorphic forms. Vol. 17 Graduate Studies in mathematics, AMS (1997).

[IS02] H. Iwaniec and P. Sarnak: Perspectives on the analytic theory of L-functions. GAFA 2000 (Tel Aviv, 1999), Geom. Funct. Anal. (2000), Special Volume, Part II, 705-741.

[KK06] H. Katsurada, Hisa-aki Kawamura: A certain Dirichlet series of Rankin-Selberg type associated with the Ikeda lifting. Department of Mathemtics Hokkaido University, series 2006, number 806

[K162] H. Klingen: Über die Werte der Dedekindschen Zetafunktion. Math. Ann. 145 (1962), 265-272.

[K190] H. Klingen: Introductory lectures on Siegel modular forms. Cambridge Studies in Advanced Mathematics, 20. Cambridge University Press, Cambridge, 1990.

[Ko80] W. Kohnen: Modular forms of half-integral weight on $\Gamma_{0}(4)$. Math. Ann. 248 (1980), 249-266

[KS89] W. Kohnen, N.-P. Skoruppa: A certain Dirichlet series attached to Siegel modular forms of degree two. Invent. Math. 95 (1989), 449-476

[Ma79] H. Maass: Über eine Spezialschar von Modulformen zweiten Grades I,II,III. Invent. Math. 52,53,53 (1979), 95-104, 249-253, 255-265.

[Mi97] S. Mizumoto: Nearly Holomorphic Eisenstein Liftings. Abh. Math. Sem. Univ. Hamburg 67 (1997), $173-194$

[Or87] T. Orloff: Special values and mixed weight triple products (with an appendix by Don Blasius). Invent. Math. 90 (1987).

[Sh73] G. Shimura: On modular forms of half-integral weight. Ann. of Math. 97 (1973), 440-481.

[Sh76] G. Shimura: The special values of the zeta functions associated with cusp forms. Comm. pure appl. Math. 29 (1976), 783-804.

[Sh94] G. Shimura: Euler products and Fourier coefficients of automorphic forms on symplectic groups. Inv. Math. 116 (1994), 531-576.

[Sh95] G. Shimura: Eisenstein series and zeta functions on symplectic groups. Inv. Math. 119 (1995), 539-584

[Si69] C.L. Siegel: Berechnung von Zetafunktionen an ganzzahligen Stellen. Nachr. Akad. Wiss. Göttingen (1969), 87-102.

[Za77] D. Zagier: Modular forms whose Fourier coefficients involve zeta-functions of quadratic fields. Modular functions of one variable, VI (Proc. Second Internat. Conf., Univ. Bonn, Bonn, 1976), pp. 105-169. Lecture Notes in Math., Vol. 627, Springer, Berlin, 1977.

[Za80] D. Zagier: Sur la conjecture de Saito-Kurokawa (d'après H. Maass). Sém. Delange-Pisot-Poitou 1979/1980, Progress in Math. 12 (1980), 371 -394

Max-Planck Institut für Mathematik, Vivatsgasse 7, 53111 Bonn, Germany

E-mail address: heim@mpim-bonn.mpg.de 